\title{
Factors Associated with Attrition from Mindfulness-Based Cognitive Therapy in Patients with a History of Suicidal Depression
}

\author{
Catherine Crane • J. Mark G. Williams
}

Published online: 11 March 2010

(C) The Author(s) 2010. This article is published with open access at Springerlink.com

\begin{abstract}
We report data from a randomised controlled trial of mindfulness-based cognitive therapy to pilot procedures for people with a history of suicidal ideation or behaviour, focusing in particular on the variables that distinguish those who complete an adequate 'dose' of treatment, from those who drop out. Sixty-eight participants were randomised to either immediate treatment with mindfulness-based cognitive therapy (MBCT) $(n=33)$ or to the waitlist $(n=36)$ arm of the trial. In addition to collecting demographic and clinical information, we assessed participants' cognitive reactivity using the means end problem-solving task, completed before and after a mood induction procedure. Ten participants dropped out of treatment, and eight dropped out of the waitlist condition. Those who dropped out of MBCT were significantly younger than those who completed treatment, less likely to be on antidepressants, had higher levels of depressive rumination and brooding and showed significantly greater levels of problem-solving deterioration following mood challenge. None of these factors distinguished participants in the waiting list condition who remained in the study from those who dropped out. Our results suggest that individuals with high levels of cognitive reactivity, brooding and depressive rumination may find it particularly difficult to engage with MBCT, although paradoxically they are likely to have the most to gain from the development of mindfulness skills if they remain in class. Addressing how such patients can be best
\end{abstract}

C. Crane $(\bowtie) \cdot$ J. M. G. Williams

Department of Psychiatry, University of Oxford,

Warneford Hospital,

Warneford Lane,

Oxford OX3 7JX, UK

e-mail: catherine.crane@psych.ox.ac.uk prepared for treatment and supported to remain in treatment when difficulties arise is an important challenge.

Keywords Mindfulness-based cognitive therapy - Attrition · Cognitive reactivity $\cdot$ Rumination $\cdot$ Dropout

\section{Introduction}

Mindfulness-based cognitive therapy (MBCT) is a manualised group-based treatment programme, which combines cognitive therapy with intensive training in mindfulness meditation, and was designed to be delivered to patients in recovery from major depression. Two previous trials of MBCT for recurrent depression have demonstrated that the treatment significantly reduces the risk of relapse over a 12-month period in those with three or more depressive episodes (Ma and Teasdale 2004; Teasdale et al. 2000), with a more recent trial demonstrating that $\mathrm{MBCT}$ has equivalent results to continuation antidepressant medication over a 1-year follow-up (Kuyken et al. 2008).

Although these trials of MBCT have based their outcomes on intention-to-treat analysis, it is notable that across the three studies, $17 \%, 16 \%$ and $15 \%$ of participants allocated to MBCT dropped out before receiving an adequate dose of treatment (four sessions), with approximately half in each case dropping out before receiving any treatment sessions at all. Although these levels of dropout compare favourably with dropout from treatment trials for patients with acute depression (e.g. Evans et al. 1992, 40\% attrition by 12 weeks for antidepressants and/or cognitive therapy; Warden et al. 2009, Star*D, 27\% attrition by 12 weeks for antidepressant treatment) and dropout from cognitive behavioural therapy (CBT) as part of routine clinical practice $(22.5 \%$ of 1,646 patients offered CBT in an 
National Health Service clinic in the UK; Westbrook and Kirk 2005) understanding the factors which influence attrition from MBCT is nevertheless important, particularly if is to be utilised with harder-to-treat clinical groups, including those with a more severe history or those with more residual symptoms, where expected levels of attrition would be higher.

We are particularly interested in issues of retention in treatment since our research is concerned with the use of mindfulness-based cognitive therapy for patients who have a history of suicidal ideation or behaviour. Although MBCT may hold promise for these patients (e.g. Williams et al. 2006), difficulties with engagement and retention in treatment can be pronounced. For example, in the POPMACT trial of brief CBT for suicide attempters, $38 \%$ of those allocated to CBT treatment failed to attend even one treatment session (Tyrer et al. 2003). Rudd et al. (1996) found a $21 \%$ drop-out rate in a treatment study of young suicidal adults. Although this appears to be comparatively low, patients were enrolled in a 2 -week intensive partial hospitalisation intervention, which is likely to have increased compliance above levels seen in outpatient studies. Finally Brown et al. (2005) managed to retain almost all the participants in their trial of cognitive therapy for suicide attempters (only two out of 60 did not attend cognitive therapy) but did so in the context of very intensive efforts including the use of study case managers. The ability to provide two verified contacts to assist with tracking and retention was included as a pre-requisite of entry to the trial, after pilot work indicated that dropout was otherwise likely to pose a significant threat to the study (Henriques, personal communication, 2004).

The three previous trials of MBCT for recurrent depression have all examined socio-demographic and clinical history variables, which might explain the causes of attrition from treatment. Two factors were found to distinguish dropouts from MBCT from those who remained in treatment: having a history of two, rather than three or more episodes of depression (associated with increased likelihood of dropout; Ma and Teasdale 2004) and having a history of attempted suicide (associated with increased likelihood of dropout; Kuyken et al. 2008). No other baseline variables distinguished the groups. Studies of meditation-based stress reduction (MBSR) programmes have also identified relatively few factors, which predict dropout. For example, Kabat-Zinn and Chapman-Waldrop (1988) reviewed adherence in a standard 8-week MBSR programme over a 2 -year period and found an average completion rate of $76 \%$, with $9 \%$ attending no sessions and a further $15 \%$ dropping out after the beginning of the programme, usually within the first few weeks (a pattern very similar to that observed in MBCT). Amongst patients referred for chronic pain, gender (being male), but not pain severity, chronicity or global distress (SCL-90), differentiated those who dropped out of treatment from those who remained. Amongst patients referred for stress-related complaints, only the obsessive-compulsive symptoms subscale of the SCL-90 differentiated groups (those higher in obsessive-compulsive symptoms being more likely to complete treatment). Similarly Carmody and Baer (2008) reported drop-out figures for participants attending MBSR classes at the University of Massachusetts Medical School in 2006 who consented to participate in a research study exploring the effects of home practice on outcome. Participants who dropped out $(5 \%, n=10)$ or failed to provide post-treatment measures $(11 \%, n=22)$ did not differ from those who completed the study protocol on the measured socio-demographic factors, nor on baseline measures of medical and psychological symptom severity, perceived stress, well being or mindfulness.

It is important to note that many of the variables that have been explored in relation to dropout concern sociodemographic factors or clinical history. Even if such variables are found to distinguish dropouts from treatment completers, they represent things which, by their very nature, cannot be changed. If, on the other hand, we could identify psychological process variables which render a person at increased risk of dropout, these might give an indication of how best to prepare patients for challenges they will face when beginning treatment. For example Lynch (2004) examined factors associated with adherence to MBSR amongst women with fibromyalgia. Sixty-nine percent of participants attended at least five out of the eight treatment sessions with only emotional role functioning predicting attendance at treatment sessions: Those participants who reported that emotional problems interfered more with their ability to function in daily life attended fewer sessions, suggesting that where problems in daily functioning are identified, these are likely to impact upon the ability to fully participate in treatment.

MBCT is designed to be delivered to patients in remission from major depression. The intention is that patients have the opportunity to practice cultivating nonjudgemental awareness in relation to relatively innocuous thoughts, feelings and body sensations, before attempting to apply this same mode of processing to negative mental content. From the first session onwards, meditation practices are used to bring participants into direct contact with their moment to moment experience in order to develop their capacities to attend to and accept their experience. However for those who may have ruminated about or tried to suppress negative thoughts and feelings over many years, the prospect of adopting such a radically different approach may be daunting. Indeed, for some, negative thoughts and feelings may be very 'close to the surface', and these patients may be immediately confronted with challenging 
mental content when they begin meditation. Salmon et al. (1998), in a review of adherence in MBSR programmes, report that one study found that chronic pain patients who experienced anxiety more somatically preferred the most 'cognitive' form of meditation (e.g. sitting), whilst those who tended to experience anxiety mainly as a cognitive phenomenon preferred more active forms of meditation (e.g. walking and yoga). This is consistent with the idea that participants may, at least initially, find it easier to engage with meditation practices that bring them into less close contact with their core aversive experiences. If this is the case, then the ability to identify people who are likely to find classes challenging and tailor the programme more specifically to their needs, or prepare them better for what they are likely to experience when they begin meditating, would be very beneficial. However none of the studies of MBCT to date have examined whether psychological process variables, which might capture such difficulties, are able to predict attrition from treatment.

Two such variables, which might predict difficulty in engaging with MBCT, are high levels of cognitive reactivity and a tendency to engage in rumination. Whilst many patients with a history of depression show evidence of cognitive reactivity - the rapid reactivation of depressogenic cognitive patterns under conditions of low mood-it appears that this cognitive profile is particularly pronounced in patients with a history of suicidality, where it extends to behavioural problems as well as to the reactivation of dysfunctional beliefs. For example Williams et al. (2005) studied the effects of induced negative mood on interpersonal problem-solving performance in participants with a history of depression with or without suicidal ideation, as well as in healthy controls. All three participant groups were indistinguishable in their problem-solving performance at baseline. However following the negative mood induction, previously suicidal participants showed a significant reduction in problem-solving effectiveness, whilst the performance of the other two groups remained relatively unchanged. The reactivation of problem-solving impairments in response to minor fluctuations in negative mood might readily undermine a patient's commitment to relatively intensive psychological treatment, and might also be a marker of more generally elevated levels of cognitive reactivity

There is growing evidence that suicidality is also associated with increased levels of rumination (see Morrison and O'Connor 2008 for a systematic review of the literature in this area). This might be important in understanding attrition for two reasons. Firstly, because rumination is likely to involve the rehearsal of depressogenic cognitions, the more an individual ruminates, the more accessible, habitual and toxic these negative cognitions are likely to become, making them more likely to emerge early during meditation, when cognitive control is relinquished. Secondly, rumination and mindful observation of experience are in some ways opposite states, so habitual ruminators are likely to find it particularly difficult to adjust to this approach, although paradoxically it might have the most to offer them.

In order to examine whether these cognitive variables might be related to attrition from treatment, we utilised data collected from a randomised controlled trial of MBCT in which we were exploring how best to adapt the programme for patients with a history of suicidal depression including those with both uni- and bipolar disorders. This trial was conducted as a feasibility and acceptability study, and the main outcomes have been reported elsewhere (Williams et al. 2008, primary outcomes; Crane et al. 2008; Hepburn et al. 2009, secondary outcomes). In this paper, we explore in detail the clinical and cognitive factors associated with attrition from treatment. Specifically we hypothesised that those who dropped out of treatment would show evidence of higher levels of cognitive reactivity, assessed by degree of deterioration in problem-solving effectiveness following induction of negative mood (Williams et al. 2005), and higher levels of rumination as assessed by the ruminative responses scale (Treynor et al. 2003). Importantly, because these data were collected from a randomised controlled trial, we were able to examine whether factors associated with dropout from MBCT also affected dropout of participants allocated to the waiting list arm, or whether they were specific to treatment.

\section{Methods}

\section{Inclusion/Exclusion Criteria}

The primary inclusion criteria for the trial were the presence of at least one past episode of MDD and a history of active suicidal ideation (suicidal thoughts including thoughts of methods of suicide) or a suicide attempt. We required individuals to be well at entry to the trial, reporting no more than 1 week of minimal symptoms of MDD in the past 8 weeks. Individuals with schizophrenia, obsessivecompulsive disorder, current eating disorder or marked alcohol or substance misuse problems (to an extent that would preclude attendance at classes) or difficulties reading, writing or speaking fluent English were excluded, as were those aged under 18 or over 65 years. Individuals with bipolar disorder were eligible to take part so long as they had not experienced a manic/hypomanic episode within the past 6 months. We did not impose restrictions concerning current medication use but individuals who had received past CBT were excluded unless they had experienced a relapse to MDD since their last session of CBT. 


\section{Recruitment}

Participants were recruited through posters, newspaper articles and features in the local media, which invited individuals who had been depressed and suicidal in the past, but who were currently feeling better, to contact the research team to take part in a clinical trial of a new psychological treatment to help people stay well. Having read the information sheet and additional information about the trial, 125 individuals expressed an interest in taking part and underwent telephone screening to assess eligibility in terms of the study inclusion/exclusion criteria outlined above. Forty-two individuals were excluded at this point due to ineligibility (20 experiencing current depression/suicidal ideation, four experiencing current mania/hypomania, nine due to recent CBT, three because they had not experienced suicidal ideation or behaviour of sufficient severity in the past and six for other reasons that would have made the classes or assessments unsuitable; e.g. Asperger syndrome and learning difficulties). Eighty-three individuals were judged eligible to take part and were invited to attend the Department of Psychiatry for a more detailed screening interview and the completion of questionnaires and cognitive tasks. Of these individuals, 11 did not attend their first assessment, two were judged to be ineligible following more detailed screening (one due to current psychotic symptoms and one due to aggression and current suicidal ideation), and two withdrew themselves from the study before completing all the assessment measures. This left 68 individuals who entered the treatment trial and were randomised to immediate or delayed treatment (see below). The flow of participants through the trial is shown in Fig. 1.

\section{Design and Procedure}

Following recruitment, participants attended the Department of Psychiatry for a detailed screening assessment. During this assessment, we obtained information on psychiatric history and current psychiatric disorders. Individuals also completed a number of questionnaires and several cognitive tasks including the means end problem-solving task (Platt et al. 1978) and a Word Fluency Task (Lezak 1976). Both these tasks were administered before and after a negative mood induction. Following completion of the first assessment, we randomly allocated eligible participants to immediate or delayed treatment. Those participants randomised to immediate treatment were invited to attend an introductory meeting with the psychologist running their classes. Those who were randomised to delayed treatment were informed that the research team would be keeping in touch with them periodically by letter during the waiting period and that they, like those receiving immediate treatment, would be asked to attend the Department of Psychiatry for a second assessment

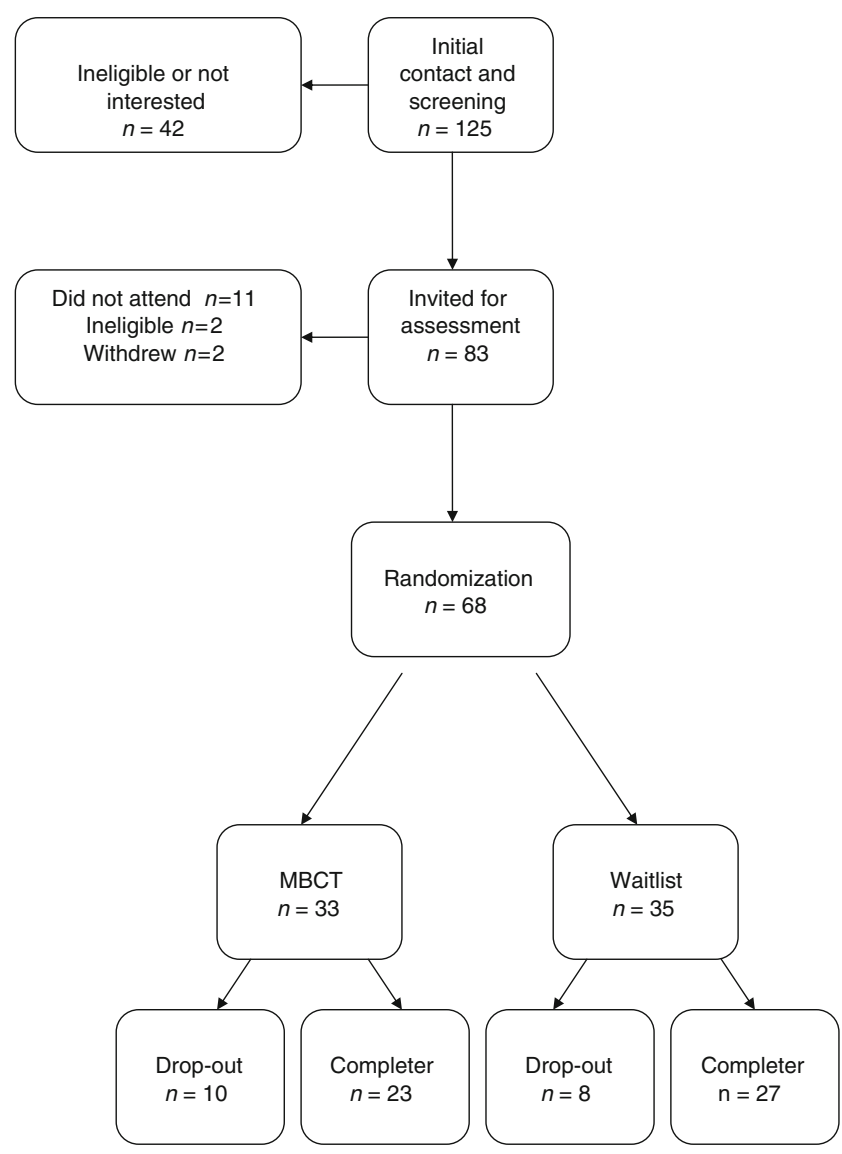

Fig. 1 Flow of participants through the trial.

session. Following the completion of the classes or waiting period, all participants who could be contacted were invited back to attend a second assessment session during which they completed the same questionnaires and cognitive tasks as at baseline. Participants' full travel expenses to attend both research assessments and treatment were reimbursed. Additionally participants received $£ 10$ per research assessment attended in recognition of the time commitment participation entailed.

\section{Retention Efforts}

Where a patient failed to attend a treatment session, therapists attempted to contact the patient by telephone, email and if necessary by letter to re-establish contact and retain the person in treatment. Where a participant failed to attend a session, we sent them materials by post, and invited them to continue practicing at home. At the end of the treatment or waiting period, extensive efforts were made to contact all participants, both those allocated to MBCT and those allocated to the waitlist arm by letter, telephone and email to schedule appointments. A dropout was defined as someone who failed to attend at least four classes for those allocated to MBCT or failed to provide any data for 
the follow-up assessment for those allocated to the waiting list condition.

\section{Randomisation}

Randomisation was stratified according to number of previous episodes of MDD $(<3$ or $3+)$ and history of suicidality (ideation or attempt). Randomisation envelopes were prepared and sealed by an individual outside the research team, and randomisation was conducted by a member of the team blind to the interpretation of the stratification numbers. Thirty three of the participants were randomised to receive immediate treatment, and 35 were randomised to receive delayed treatment. Participants were informed of the outcome of randomisation by letter following completion of all initial assessments.

\section{Measures}

Clinical Interview At the first assessment, participants were screened using the Mini International Neuropsychiatric Interview (MINI; Sheehan et al. 1998) and the Structured Clinical Interview for DSM-IV Axis II (SCID-II) borderline personality disorder module (First et al. 1997). The MINI is a structured interview, which provides DSM-IV diagnoses of Axis I psychiatric disorders, and has excellent reliability when compared to diagnoses derived from the SCID (Sheehan et al. 1998).

Beck Depression Inventory (Beck et al. 1996) The Beck Depression Inventory (BDI-II) is a well-established measure of depressive symptomatology that contains 21 groups of statements, referring to the presence of symptoms of depression over the preceding 2 weeks.

Beck Anxiety Inventory (Beck and Steer 1990) The Beck Anxiety Inventory (BAI) is a 21-item self-report questionnaire that measures the common symptoms of anxiety. The scale is reliable and valid and is able to differentiate anxiety from depression.

Ruminative Responses Subscale (Nolen-Hoeksema and Morrow 1990; Treynor et al. 2003) The Ruminative Responses Subscale (RRS) consists of three subscales assessing how people react when feeling low or depressed: depressionrelated rumination (12 items, e.g. 'think about feelings of achiness and fatigue', 'think about how hard it is to concentrate'), brooding (five items, described by the authors as 'moody pondering' e.g. "think 'why can't I handle things better?"') and reflection (five items referring to neutrally valenced pondering, e.g. 'analyse recent events to try and understand why you are depressed'; see Treynor et al. 2003 for factor analysis).
Cognitive Reactivity Assessment

Mood Induction In order to assess the effects of negative mood on problem solving and verbal fluency, we administered a combined mood induction. Participants listened to Prokofiev's 'Russia under the Mongolian Yoke' (Alexander Nevsky Suite) played at half speed over a period of $8 \mathrm{~min}$ whilst reading a set of 32 negative Velten statements (e.g. 'There are things about me that I don't like' or 'I just want to curl up and go to sleep'). Mood was measured at the beginning at end of the mood induction and the beginning and end of the experiment using VAS scales on which participants indicated how 'sad' and 'happy' they felt at that moment.

Means End Problem-Solving Task (Platt et al. 1978) The means end problem-solving task (MEPS) assesses participants' ability to generate effective solutions to interpersonal problems. In order to assess problem-solving ability, participants are presented with a problem and a desired end-point and were given $1 \mathrm{~min}$ to describe the most effective way of getting from the beginning to the end of the scenario. For the current study, we used 12 problem-solving scenarios (to enable assessment of problem solving through three scenarios pre- and post-mood induction, both before and after treatment or waitlist), including both the traditional scenarios from Platt and Spivak (losing a watch, making friends in a new neighbourhood, having difficulty with a supervisor at work, starting a relationship, resolving problems with friends and dealing with difficulties in a romantic relationship) and six comparable scenarios developed for the current study (finding a new job, helping a friend who does not seem to be their usual self, dealing with problems with an electricity supplier, resolving problems caused by troublesome young people in the neighbourhood, maintaining a relationship with a platonic friend who has romantic feelings and coping with short-term financial difficulties). Scenarios were grouped into four blocks to ensure that each block contained a mixture of themes (e.g. work, romantic relationships, friendships, neighbourhood and practical problems). The order of presentation of these blocks was counterbalanced across participants, and analysis indicated no significant effects of order of presentation on effectiveness ratings.

Previous research has shown that it is problem-solving effectiveness, rather than number of relevant means, which differentiates participants with a history of suicidal ideation from those with a history of non-suicidal depression (Williams et al. 2005). For the purposes of the current study, effectiveness was rated on a scale ranging from 1 (not at all effective) to 7 (extremely effective) by a rater blind to participant allocation (immediate treatment versus waitlist) and to whether the each MEPS solution had been completed before or after the mood induction procedure. A 
$10 \%$ sample of problem solutions was rated by a second independent rater, yielding a weighted kappa of 0.76 , indicating good agreement.

Verbal Fluency Task (Lezak 1976) The Verbal Fluency Task was administered before and after the mood induction, to provide a control for the effects of mood on general speed of cognitive processing. Participants were instructed to say aloud as many words as they could think of beginning with each of two letters FA, SD, RT or DM in $30 \mathrm{~s}$ without repeating words or using different versions of the same word. The letter sets were counterbalanced across participants and time of assessment (before and after mood induction and before and after the treatment or waitlist period). The total number of valid words generated by participants was recorded on each occasion.

\section{MBCT Classes}

All participants who were randomised to MBCT were offered a pre-class interview with the instructor who would be leading their class. This interview took approximately $1 \mathrm{~h}$ and covered expectations for the classes and practical matters. The MBCT course itself was delivered in eight sessions plus an all-day practice over a period of 9 weeks (nine attendances in total). The treatment closely followed the manual of Segal et al. (2002) with the modification that participants completed an exercise in which they developed a crisis plan in sessions 6 and 7 .

\section{Results}

\section{Demographics}

Sixty-eight participants (26 men and 42 women) were recruited to the study. The mean age of the sample was 42 years $(\mathrm{SD}=10.92$, range $22-64)$. The sample was generally well educated. Six participants (9\%) had left school without any qualifications, seven (10\%) had taken school leaving exams aged 16, 13 (19\%) had completed further education, $22(32 \%)$ had a degree or equivalent, and $20(29 \%)$ had a postgraduate qualification. Across the sample as a whole, 29 (43\%) individuals were single, 20 $(29 \%)$ were married, eight (12\%) were cohabiting, and nine $(13 \%)$ were separated, divorced or widowed. For two individuals data on marital status was missing.

\section{Clinical History}

Episodes of MDD The median number of episodes of depression reported by participants was 4 (range 1-30). Ten individuals reported chronic symptoms, with episodes of depression that were numerous but where the precise number, could not be determined. The mean reported age of onset of depressive symptoms was 20 years, the modal age of onset was 15 years, with approximately $40 \%$ of participants reporting that their first onset of depressed mood occurred in childhood or adolescence $(<16$ years).

Suicide Attempts Twenty-six individuals (38\%) had made one or more suicide attempt in the past. Amongst attempters, the median number of suicide attempts was 1 (range 1-16), with 11 individuals having attempted suicide only once, 11 having attempted suicide on between two and five occasions and four having made more than five suicide attempts.

Medication Usage Thirty-four individuals were taking psychotropic medication at entry to the trial (data on medication was missing for two participants). Thirty individuals were taking one or more antidepressant drug, two were taking tranquilisers, five were taking antipsychotic medication, and five were taking lithium or another mood stabiliser. Several participants were taking multiple medications at entry.

Psychotherapy Fifty-four participants reported having received some prior psychotherapy, psychological support or counselling for depression. Seventeen participants reported past cognitive behaviour therapy (all with subsequent relapse to MDD). However in many cases, the treatment received appeared to have been groupadministered rather than individual and was often of limited duration (four to six sessions).

Hospitalisation Fifteen participants had received inpatient treatment for depression or mania in the past (seven with a single admission, two with two admissions, three with three admissions and three with five or more admissions).

Baseline Comparisons Between Individuals Allocated to Immediate and Delayed Treatment

Clinical and Demographic Factors There were no significant differences between individuals allocated to immediate and to delayed treatment in age, educational level, proportion of participants who had attempted suicide, number of episodes of MDD, age of onset of MDD, prior inpatient treatment (yes/no), prior psychotherapy, or prior CBT (all $p$ 's $>0.50$ ). The immediate and delayed treatment group also did not differ in the proportion of participants taking antidepressant medication at entry $\left(\chi^{2}=0.16, d f=1\right.$, $p>0.63)$. The numbers taking tranquilisers, anti-psychotics 
and mood stabilisers (e.g. lithium) were very small but distributed equally across the immediate and delayed groups (1:1, 2:3 and 2:3 respectively).

Questionnaires The mean score on the BDI-II at baseline was $12.85(\mathrm{SD}=10.89)$, approaching the cutoff for mild depressive symptoms (score of 13). The mean score on the BAI was $11.56(\mathrm{SD}=9.84)$. Individuals allocated to immediate treatment and those allocated to delayed treatment did not differ significantly from one another at baseline on the BDI-II or BAI scores. There were also no differences between the groups on the BSSw or the Response Styles Questionnaire (RSS) total, depressive rumination, brooding and reflection subscales (all $p$ 's $\geq 0.20$ ).

Mood Induction Mood Ratings Visual analogue mood ratings were taken at four time points during the experimental session (start, pre-mood induction, postmood induction and end). Repeated-measures analysis of variance (ANOVA) indicated a main effect of time on ratings of sadness, $F(3,198)=53.91, p<0.001$ and happiness $F(3,198)=28.16, p<0.001$, and comparisons of the means indicated that the mood induction was effective in producing increases in sadness and decreases in happiness. There was no significant time $\times$ group interaction, indicating that the mood induction had a similar effect on those subsequently allocated to immediate treatment and those in the waitlist condition.

Means End Problem-Solving Task Repeated-measures analysis of variance was used to examine the effect of the negative mood induction on problem-solving effectiveness ratings, comparing individuals allocated to immediate and delayed treatment. There was a trend towards an effect of time on effectiveness of problem solving, with participants tending to provide less effective solutions after the mood induction, $F(1,65)=3.58$, $p=0.06$, replicating previous findings in suicidal populations (e.g. Williams et al. 2005). There was no interaction between time and group, indicating that individuals allocated to immediate versus delayed treatment were well matched in cognitive reactivity at baseline.

Word Fluency Analysis indicated no significant main effect of time, $F(1,66)=2.67, p=0.11$, and no time by group interaction. However there was a significant main effect of group, $F(1,66)=4.93, p<0.05$, with participants allocated to immediate treatment having superior word fluency at baseline. These results suggest that the trend towards a deterioration in problem-solving effectiveness was not simply the result of decreased verbal fluency following mood induction.
Levels of Treatment Adherence

One of the primary aims of this trial was to determine factors affecting the ability of previously suicidal individuals to participate in MBCT. Participants could attend a maximum of nine sessions of MBCT (eight evening classes, plus one all-day practice). Of the 33 individuals randomised to immediate treatment, $23(70 \%)$ attended four or more sessions of MBCT (defined as a minimum adequate dose), and ten (30\%) dropped out. The mean number of sessions attended by those receiving an adequate dose of treatment (i.e. four or more sessions) was $8.09(\mathrm{SD}=1.20)$ as compared to 1.50 $(\mathrm{SD}=1.23)$ amongst those who did not, indicating that consistent with previous studies, individuals who dropped out tended to do so early in treatment, and those who remained in treatment had very high levels of adherence.

We first consider baseline factors that differentiated individuals who were allocated to immediate treatment and dropped out from those who completed treatment. We then go on to examine whether factors differentiating MBCT treatment dropouts from treatment completers also differentiate waitlist dropouts from individuals in the waiting list group who remained in the study until the second assessment. The absence of equivalent differences between waitlist dropouts and individuals in the waiting list group who remained in the trial would lend support to the suggestion that factors distinguishing treatment dropouts from adherers are related to difficulty with participation in the treatment itself, rather than to other non-specific factors affecting participation in a clinical trial.

\section{Factors Related to Attrition from MBCT}

Demographic Variables Five men and five women dropped out, and 11 men and 12 women completed the treatment, indicating no significant effect of gender. However individuals who dropped out of treatment were significantly younger than those who remained in treatment, $U=55.00, Z=-2.35, p=0.02$.

Clinical Variables There was no significant difference between those who dropped out and those who completed treatment in the number of prior episodes of major depression, a history of manic or hypomanic episodes, the proportion with lifetime or current alcohol or substance abuse or dependence or borderline personality disorder. A greater proportion $(n=6,60 \%)$ of treatment dropouts had attempted suicide as compared to treatment adherers $(n=7$, $30 \%$ ) but this difference in proportions was not statistically significant. Individuals who dropped out were significantly less likely to be taking antidepressants $(n=1,10 \%)$ at 
baseline than those who remained in treatment $(n=11$, $48 \%$ ), Fisher's exact test $p=0.05$. The low frequencies of other medications precluded statistical analysis but there were no apparent differences between groups. Treatment dropouts did not differ significantly from treatment completers in the proportion who had previously had psychotherapy $(n=8,80 \%$ versus $n=17,52 \%)$. There was no difference between the groups in the proportions who had received cognitive therapy ( $n=2,20 \%$ versus $n=6,26 \%)$. Finally there was no significant difference between the dropouts and adherers in terms of hospital admission for psychiatric problems ( $n=1,10 \%$ versus $n=4,17 \%)$.

Questionnaire Measures No differences between dropouts and completers were observed on baseline measures of depression (BDI-II) or anxiety (BAI). However individuals who dropped out of treatment had a significantly higher mean score on the measures of depressive rumination, $t(31)=2.32, p=0.03$, and brooding, $t(31)=$ $3.48, p=0.002$, although they did not differ on the RSS reflection subscale, $p>0.30$.

Assessment of Cognitive Reactivity A repeated-measures ANOVA comparing the effect of negative mood induction on problem-solving effectiveness in individuals who completed treatment and those who dropped out revealed a main effect of time at trend level, $F(1,31)=2.85, p=0.10$, and a significant time $\times$ group interaction, $F(1,31)=7.87$, $p=0.009$. Post hoc tests indicated that whilst individuals who subsequently dropped out of treatment deteriorated significantly (by approximately $20 \%$ ) in problem-solving effectiveness from pre- to post-mood induction $(p=0.01)$, those who subsequently remained in treatment showed no significant change (see Fig. 2). There were no significant main effects of group, nor of time $\times$ group interactions on the measures of mood or verbal fluency taken before and after mood induction. This suggests that the greater vulnerability to mood-related deteriorations in problem solving amongst those who dropped out of treatment were unlikely to be simply the result of more marked shifts in mood, or general deteriorations in performance on cognitive tasks.

\section{Logistic Regression}

In order to determine whether the different factors identified from univariate analyses independently contributed to the prediction of dropout from treatment, we conducted a binary logistic regression analysis with age, antidepressant use, problem-solving change score and brooding entered simultaneously into the model as independent variables. Brooding was selected for entry rather than entering both brooding and depressive rumination as the two measures

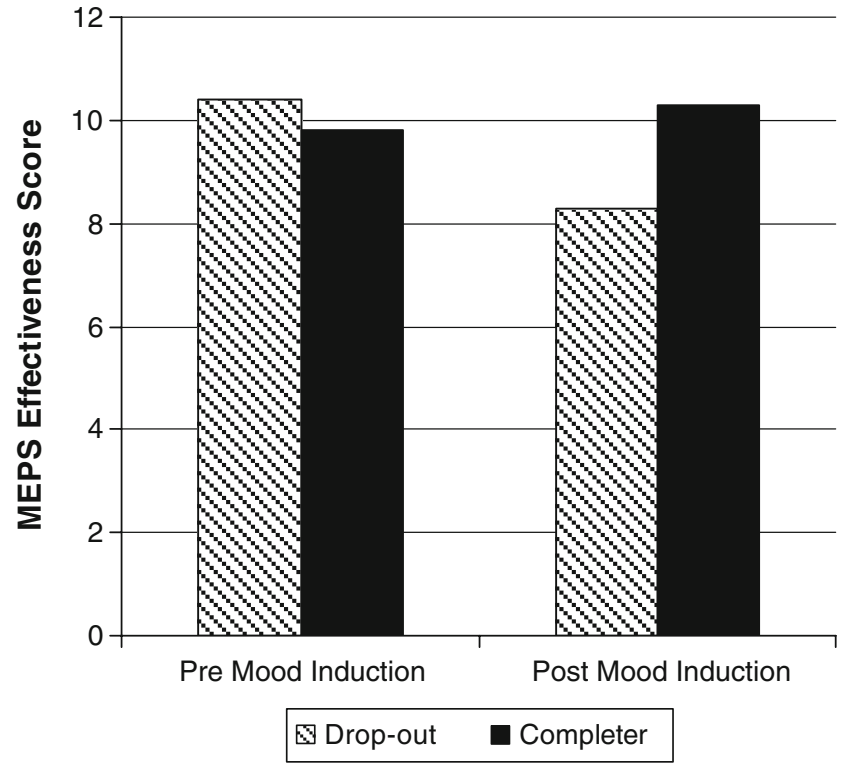

Fig. 2 Effect of mood induction on problem-solving effectiveness in treatment dropouts and completers.

were highly correlated, $r(33)=0.57, p=0.001$, and brooding more strongly distinguished the groups in the univariate analyses. The regression model was highly significant, $\chi^{2}(4)=22.61, p<0.001$, correctly classifying $90 \%$ of participants as completers or dropouts. Both effectiveness change, Wald $=4.98, \operatorname{Exp}(B)=0.42, d f=1, p=0.03$ and brooding, Wald $=3.80, \operatorname{Exp}(B)=0.61, d f=1, p=0.05$ were significant independent predictors of dropout. Age and antidepressant use did not contribute significantly to the model in the context of the other variables.

\section{Factors Related to Dropout from the Waitlist Condition}

To explore whether the differences observed between those who remained in treatment and those who dropped out related to the acceptability of treatment, rather than participation in the research elements of the trial, we compared the variables found to be related to attrition from treatment between individuals who were allocated to the waiting list condition who did, $n=27$, or did not $n=8$ attend the follow-up assessment session. Due to the very small numbers being compared, these analyses should clearly be interpreted tentatively.

Comparisons Non-parametric comparisons between the groups revealed no significant differences on the RSS rumination $(p=0.59)$ and brooding $(p=0.35)$ subscales between those participants who remained in the waitlist arm of the trial and those who dropped out. Similarly a repeated-measures ANOVA indicated that whilst effectiveness deteriorated somewhat in both groups from pre- to post-mood induction, this effect did not reach statistical 
significance, $F(1,32)=2.13, p=0.15$. There was no main effect of group and no time $\times$ group interaction. However inspection of the mean scores pre- and post-mood induction revealed that in the waiting list condition, the effect of the mood induction was slightly more pronounced in those who remained in the study.

\section{Discussion}

Although the majority of previous trials of MBCT and MBSR have compared those who dropped out to those who completed treatment in terms of baseline characteristics, very few factors have been identified that predict attrition to date. Additionally, where factors have been identified, these usually relate to socio-demographic characteristics or clinical history rather than to proximal cognitive factors, which might guide the modification of treatment for those at risk of dropout. In this paper, we explored predictors of attrition in patients with a history of suicidal depression randomly allocated to treatment with $\mathrm{MBCT}$ as part of a feasibility trial exploring the usefulness of MBCT for patients with a history of suicidal ideation or behaviour. Since these patients may be particularly at risk of dropout from treatment, identification of variables which might predict attrition and which might be amenable to compensatory efforts on the part of the therapist is crucial.

Almost one third of patients $(30 \%)$ dropped out of MBCT, having attended fewer than four classes, a higher percentage than observed in previous trials of MBCT that had enrolled previously depressed patients unselected for history of suicidality (e.g. Ma and Teasdale 2004; Kuyken et al. 2008; Teasdale et al. 2000). More consistent with previous findings, however, was the observation that where dropout occurred, it tended to do so very early in treatment (in most cases before treatment had started, or after only one session). We identified a number of variables that predicted attrition: levels of depressive rumination and brooding (higher levels associated with greater dropout), age (those who were young relative to the average for participants in class were more at risk), antidepressant medication (those not taking medication were more at risk) and increased cognitive reactivity to sad mood (those who showed greater deteriorations in problem-solving performance were more likely to drop out). Logistic regression analysis, to explore the predictive capacity of each of the variables identified in the univariate analysis in parallel, indicated that both brooding and cognitive reactivity had significant independent effects on dropout. None of the factors that differentiated MBCT treatment completers from dropouts distinguished completers and dropouts in the waitlist arm of the trial, lending some support to the suggestion that these variables may relate more closely to difficulties with the experience (or prospect) of treatment itself than to more generic aspects of compliance with the research protocol.

Given the relatively small sample size, any conclusions must be tentative. However our observation that individuals who have high levels of cognitive reactivity find it more difficult to engage with MBCT does makes theoretical sense. Cognitive reactivity describes the process by which small changes in mood rapidly activate depressogenic cognitions and behavioural deficits. Patients with high levels of cognitive reactivity are likely to be more frequently confronted with distressing thoughts and feelings and less able to respond in a way which is adaptive, when they occur. Mindfulness training requires direct and sustained contact with all experience and so is likely to bring these aversive experiences into sharp relief, earlier in the process of treatment than might be the case for patients with less reactive profiles. Similarly those who typically respond to unwanted experiences with brooding are invited to relinquish their habitual responses from the outset of treatment, before they have had the opportunity to experience the benefits of this approach directly. Research in substance abuse populations shows that low levels of psychological distress tolerance predict early dropout from treatment (Daughters et al. 2005), and it would be interesting in future research to explore the extent to which the same is true of MBCT.

The identification of people who are likely to have difficulties engaging with treatment may be beneficial, and whilst it is not practical to conduct mood inductions in routine clinical practice, careful questioning of participants may be able to identify those whose responses to low mood are characterised by cognitive reactivity and impairments in functioning. Indeed the measure of emotional role functioning, which differentiated dropouts from treatment completers in Lynch (2004), may capture the type of deterioration in problem solving identified in the current study since it is described as a measure of 'perceived limitations in role functioning due to emotional factors' (p. 47), e.g. the inability to functioning effectively when experiencing emotional distress.

It is our observation that if mindfulness teachers are able, during their pre-class interview, to discuss in detail the rationale for treatment (and why reducing levels of rumination is likely to be helpful), establish realistic expectations (that early mindfulness practices will be challenging and may include the experience of distressing emotions) and provide some strategies for how to respond if such emotions do arise (for example discussing grounding techniques, encouraging contact with the teacher between classes and emphasising the need for perseverance); these can enhance motivation and increase persistence when difficulties 
do arise. Most mindfulness teachers are likely to cover some or all of this information in their pre-class interviews. However identifying the people for whom this is likely to be most critical and monitoring their progress in early classes may help.

Two other factors were identified in univariate analyses, and given the exploratory nature of the study, we discuss them briefly. First, those who dropped out were significantly less likely to be on antidepressants that those who completed treatment. All participants had a history of depression, the majority with several prior episodes (median of 4). Since maintenance antidepressants are recommended for all people with such a history (NICE 2009), it is possible that the fact that these patients were not on antidepressants is a sign of lack of engagement with treatment in general. Alternatively it is possible that antidepressant medication stabilises people, enabling them to then benefit more from MBCT, at least at the outset. Further research examining differential responding to MBCT in patients on and off antidepressant medication, matched for other clinical history variables, would shed light on this issue.

Age was the final factor which distinguished the dropouts from the completers. A similar finding has been observed for dropout from personality disorder services (Crawford et al. 2009) with whom our population of previously suicidal patients may have some overlap. One possibility is that individuals who were younger than average felt less able to relate to other members of the group, or felt themselves to be more of an outsider. The support provided by the group and the realisation of not being alone in suffering from depression have been repeatedly identified as core to the experience of MBCT in qualitative studies (e.g. Mason and Hargreaves 2001; Allen et al. 2009). It is possible that participants who are much younger than average have less of a sense of shared experience with other group members. They may also have a shorter history of depressive illness and hence be less motivated to engage in an intensive treatment intervention. However it is possible that the effect relates to a few younger participants who differed in some other respect, not identified here.

The most obvious limitation of our study is the small sample size on which comparisons between dropouts and treatment completers are based. This is a limitation which faces most analyses of dropout from treatment, and may account for the fact that often few significant differences are observed. Indeed only because there was relatively high dropout in this trial were such comparisons possible. In view of this, our results must be interpreted tentatively, as suggestions for future research rather than definite prognostic indicators. Additionally these data were obtained from a clinical trial of MBCT rather than from routine clinical practice. The disadvantage is that some of the factors that might be expected to relate to attrition, such as variations in general motivation to obtain treatment or household income (e.g. Warden et al. 2009), might be less relevant, since expenses to attend the research trial were reimbursed and participants were relatively highly motivated at the outset, in most cases having referred themselves to the trial directly. However, if such factors are removed or diminished in importance, this may allow for the identification of other variables, which would otherwise be missed.

Despite these caveats, we do believe that the results are of interest. Firstly, they suggest the existence of variables which relate meaningfully to risk processes and which simultaneously influence engagement in treatment. In this trial, participants with the highest levels of cognitive vulnerability were most likely to drop out of treatment before receiving an adequate dose. It is therefore likely to be essential to target these risk processes and the impact that they have on engagement with treatment, if the efficacy of treatments is to be enhanced. Secondly, this study suggests that the identification of proximal psychological variables, which predict dropout, is possible, although few studies look beyond clinical and demographic variables when examining attrition.

In our current trial for preventing suicidal depression, we give particular attention to addressing the factors that might predict dropout by giving an extended pre-class interview to the most vulnerable participants, and the early indications are that the proportion of those dropping out has fallen markedly as a result. We encourage other researchers to consider exploring factors associated with attrition in more detail, since the findings have not only direct clinical implications but also the potential to tell us much about the processes involved in the acquisition of mindfulness skills.

Acknowledgements We gratefully acknowledge the contribution of our colleagues Melanie Fennell, Thorsten Barnhofer, Danielle Duggan and Silvia Hepburn to the design and conduct of the trial from which the data reported here were extracted. We would also like to thank our participants who generously gave their time to assist with the research.

Open Access This article is distributed under the terms of the Creative Commons Attribution Noncommercial License which permits any noncommercial use, distribution, and reproduction in any medium, provided the original author(s) and source are credited.

\section{References}

Allen, M., Bromley, A., Kuyken, W., \& Sonnenberg, S. J. (2009). Participants' experiences of mindfulness-based cognitive therapy: "It changed me in just about every way possible". Behavioural and Cognitive Psychotherapy, 34, 413-430.

Beck, A. T., \& Steer, R. A. (1990). Beck anxiety inventory manual. San Antonio: Psychological.

Beck, A. T., Steer, R. A., \& Brown, G. K. (1996). Manual for the BDI-II. San Antonio: Psychological. 
Brown, G. K., Ten Have, T., Henriques, G. R., Xie, S. X., Hollander, J. E., \& Beck, A. T. (2005). Cognitive therapy for the prevention of suicide attempts: A randomized controlled trial. JAMA, 294, $563-570$

Carmody, J., \& Baer, R. A. (2008). Relationship between mindfulness practice and levels of mindfulness, medical and psychological symptoms and well-being in a mindfulnessbased stress reduction program. Journal of Behavioural Medicine, 31, 23-33.

Crane, C., Barnhofer, T., Duggan, S. D., Hepburn, S. R., Fennell, M. J. V., \& Williams, J. M. G. (2008). Mindfulness-based cognitive therapy and self-discrepancy in recovered depressed patients with a history of suicidality. Cognitive Therapy \& Research, 32, 775-787.

Crawford, M. J., Price, K., Gordon, F., Josson, M., Taylor, B., Bateman, A., et al. (2009). Engagement and retention in specialist services for people with personality disorder. Acta Psychiatrica Scandinavica, 119, 304-311.

Daughters, S. B., Lejuez, C. W., Bornovalova, M. A., Kahler, C. W., Strong, D. R., \& Brown, R. A. (2005). Distress tolerance as a predictor of early treatment dropout in a residential substance abuse treatment facility. Journal of Abnormal Psychology, 114, 729-734.

Evans, M. D., Hollon, S. D., De Rubeis, R. J., Piasecki, J. M., Grove, W. M., Garvey, M. J., et al. (1992). Differential relapse following cognitive therapy and pharmacotherapy for depression. Archives of General Psychiatry, 49, 802-808.

First, M. B., Gibbon, M., Spitzer, R. L., \& Williams, J. B. W. (1997). Structured clinical interview for DSM-IV axis-II personality disorders (SCID-II). Washington: American Psychiatric Press.

Hepburn, S. R., Crane, C., Barnhofer, T., Duggan, D. S., Fennell, M. J. V., \& Williams, J. M. G. (2009). Mindfulness-based cognitive therapy may reduce thought suppression in previously suicidal participants: Findings from a preliminary study. British Journal of Clinical Psychology, 48, 209-215.

Kabat-Zinn, J., \& Chapman-Waldrop, A. (1988). Compliance with an outpatient stress reduction program: Rates and predictors of program completion. Journal of Behavioral Medicine, 11, 333-352.

Kuyken, W., Byford, S., Taylor, R. S., Watkins, E., Holden, E., White, K., et al. (2008). Mindfulness-based cognitive therapy to prevent relapse in recurrent depression. Journal of Consulting and Clinical Psychology, 76, 966-978.

Lezak, M. D. (1976). Neuropsychological assessment. New York: Oxford.

Lynch, G. V. (2004). Patient variables associated with treatment completion in mindfulness meditation-based stress reduction (MBSR) treatment for fibromyalgia: implications for prescriptive matching and participation enhancement. Unpublished doctoral dissertation, University of Louisville, Kentucky, USA.

Ma, S. H., \& Teasdale, J. D. (2004). Mindfulness-based cognitive therapy for depression: Replication an exploration of differential relapse prevention effects. Journal of Consulting \& Clinical Psychology, 72, 31-40.

Mason, O., \& Hargreaves, I. (2001). A qualitative study of mindfulness-based cognitive therapy for depression. British Journal of Medical Psychology, 74, 197-212.

Morrison, R., \& O'Connor, R. C. (2008). A systematic review of the relationship between rumination and suicidality. Suicide \& Life Threatening Behaviour, 38, 523-538.

NICE. (2009). Depression: The treatment and management of depression in adults (update). UK: National Institute for Health and Clinical Excellence.
Nolen-Hoeksema, S., \& Morrow, J. (1990). A prospective study of depression and post-traumatic stress symptoms after a natural disaster: The 1989 Lona Prieta earthquake. Journal of Personality and Social Psychology, 61, 115-121.

Platt, J. J., Spivak, G., \& Bloom, W. (Eds.). (1978). Manual for the Means-End Problem Solving (MEPS) procedure: A measure of interpersonal problem-solving skill. Philadelphia: Hahnemann Medical College Hospital.

Rudd, D. M., Rajab, M. H., Orman, D. T., Stulman, D. A., Joiner, T., \& Dixon, W. (1996). Effectiveness of outpatient intervention targeting suicidal young adults: Preliminary results. Journal of Consulting and Clinical Psychology, 64, 170-190.

Salmon, P. G., Santorelli, S. F., \& Kabat-Zinn, J. (1998). Intervention elements promoting adherence to mindfulness-based stress reduction programs in the clinical behavioral medicine setting. In S. A. Shumaker, E. B. Schron, J. K. Ockene, \& W. L. McBee (Eds.), The handbook of health behavior change (pp. 239-266). New York: Springer.

Segal, Z. V., Williams, J. M. G., \& Teasdale, J. V. (2002). Mindfulness-based cognitive therapy for depression: A new approach for preventing relapse. New York: The Guilford.

Sheehan, D. V., Lecrubier, Y., Sheehan, K., Harnett, A. P., Janava, J., Weiller, E., et al. (1998). The Mini-International Neuropsychiatric Interview (M.I.N.I): The development and validation of a structured psychiatric interview for DSM-IV and ICD-10. Journal of Clinical Psychology, 59, 22-33.

Teasdale, J. D., Segal, Z. V., Williams, J. M. G., Ridegeway, V. A., Soulsby, J. M., \& Lau, M. A. (2000). Prevention of relapse/ recurrence in major depression by mindfulness-based cognitive therapy. Journal of Consulting and Clinical Psychology, 68, 615623.

Treynor, W., Gonzales, R., \& Nolen-Hoeksema, S. (2003). Rumination reconsidered: A psychometric analysis. Cognitive Therapy and Research, 27, 247-259.

Tyrer, P., Thompson, S., Schmidt, U., Jones, V., Knapp, M., Davidson, K., et al. (2003). Randomized controlled trial of brief cognitive behaviour therapy versus treatment as usual in recurrent deliberate self harm: The POPMACT study. Psychological Medicine, 33, 969-976.

Warden, D., Rush, J. A., Wiseniewski, S. R., Lesser, I. M., Thase, M. E., Balasurbramani, G. K., et al. (2009). Income and attrition in the treatment of depression: A STAR*D report. Depression and Anxiety, 26, 622-633.

Westbrook, D., \& Kirk, J. (2005). The clinical effectiveness of cognitive behaviour therapy: Outcome for a large sample of adults treated in routine clinical practice. Behaviour Research and Therapy, 43, 1243-1261.

Williams, J. M. G., Barnhofer, T., Crane, C., \& Beck, A. T. (2005). Problem solving deteriorates following mood challenge in formerly depressed patients with a history of suicidal ideation. Journal of Abnormal Psychology, 114, 421-431.

Williams, J. M. G., Duggan, D. S., Crane, C., \& Fennell, J. M. V. (2006). Mindfulness-based cognitive therapy for prevention of recurrence of suicidal behaviour. Journal of Clinical Psychology, 62, 201-210.

Williams, J. M. G., Alatiq, Y., Crane, C., Barnhofer, T., Fennell, M. J. V., Duggan, D. S., et al. (2008). Mindfulness-based cognitive therapy (MBCT) in bipolar disorder: Preliminary evaluation of immediate effects on between-episode functioning. Journal of Affective Disorders, 107, 275-279. 